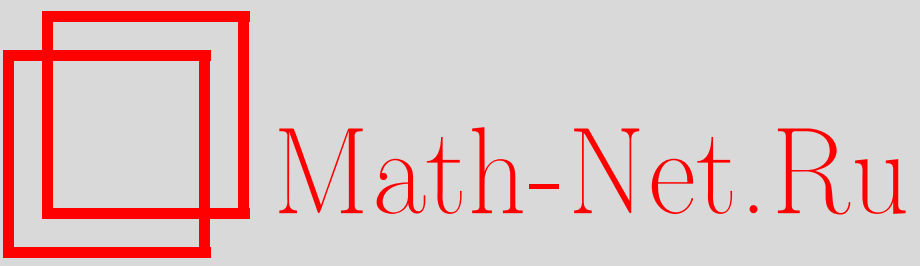

А. А. Боровков, Колмогоров и граничные задачи теории вероятностей, УМH, 2004, том 59, выпуск 1, 91-102

DOI: https://doi.org/10.4213/rm702

Использование Общероссийского математического портала Math-Net.Ru подразумевает, что вы прочитали и согласны с пользовательским соглашением

http://www.mathnet.ru/rus/agreement

Параметры загрузки:

IP : 54.224 .60 .19

26 апреля 2023 г., 16:21:39 


\title{
КОЛМОГОРОВ И ГРАНИЧНЫЕ ЗАДАЧИ ТЕОРИИ ВЕРОЯТНОСТЕЙ
}

\author{
А. А. Боровков
}

\begin{abstract}
Работа состоит из двух частей. В первой прослеживается история развития граничных задач теории вероятностей, в котором Андрей Николаевич принимал самое активное участие. Излагается найденный недавно новый подход к решению граничных задач для случайных блужданий, удовлетворяющих условию Крамера. Он является более общим, простым и наглядным, чем аналитический метод, предложенный автором в $60-\mathrm{x}$ годах. Построение такого более общего альтернативного подхода Андрей Николаевич считал крайне желательным, так как чисто аналитический подход представлялся ему не вполне адекватньм существу дела.

Во второй части работы излагаются основные пределные теоремы в граничных задачах для случайных блужданий, не удовлетворяющих условию Крамера. Приводится ряд новых резултатов, полученных в последнее время.

Библиография: 20 названий.
\end{abstract}

\section{СОДЕРЖАНИЕ}

1. Граничные задачи при выполнении условия Крамера

2. О распределении максимума сумм при невыполнении условия Кра-

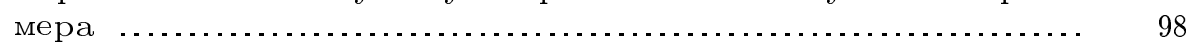

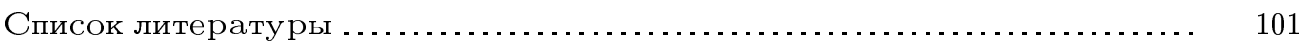

Из многих направлений деятельности А. Н. Колмогорова я остановлюсь на тех, которые в свое время оказали на меня значительное влияние и которые продолжают развиваться вплоть до сегодняшнего дня. Эти направления можно объединить одним названием "граничные задачи теории вероятностей", так как все они в той или иной мере связаны с пересечением заданных гранищ траекторией случайного блуждания.

\section{1. Граничные задачи при выполнении условия Крамера}

Под случайным блужданием мы будем понимать главным образом последовательность $\left\{S_{k}\right\}$ сумм $S_{k}=\sum_{i=1}^{k} \xi_{i}$ независимых случайных величин $\xi_{1}, \xi_{2}, \ldots$ Первой работой Андрея Николаевича в области граничных задач можно считать, по-видимому, его фундаментальную работу [1] о законе повторного логарифма (последний несомненно относится к граничным задачам, так как имеет дело с числом пересечений траекторией $\left\{S_{k}\right\}$ заданных границ). В этой его работе, как и во многих других, 
важны не только сами результаты, которые определяли развитие новых направлений, но и методология, как правило, точно адекватная существу дела. Вспомогательные результаты, которые он использовал, часто оказывались не менее важными для последуюшего развития, чем основные. Так, в названной работе о законе повторного логарифма были получены одни из первых результатов о вероятностях больших уклонений. Обозначим

$$
\sigma_{j}^{2}=\mathrm{E} \xi_{j}^{2}, \quad B_{n}^{2}=\sum_{j=1}^{n} \sigma_{j}^{2}
$$

Тогда в предположениях

$$
\mathrm{E} \xi_{n}=0, \quad B_{n} \rightarrow \infty, \quad \xi_{n}=o\left(\frac{B_{n}}{\sqrt{\ln \ln B_{n}}}\right), \quad \frac{x}{B_{n}} \rightarrow \infty, \quad x=o\left(B_{n}^{2}\right)
$$

было установлено, что

$$
\ln \mathrm{P}\left(S_{n}>x\right) \sim-\frac{x^{2}}{2 B_{n}^{2}} .
$$

Такого рода утверждения (об асимптотике логарифма изучаемых вероятностей) называют грубьми теоремами о больших уклонениях. В последуюшие годы исследования асимптотики $\mathrm{P}\left(S_{n}>x\right)$ в области больших уклонений достигли большой точности и обшности. Это отдельное большое направление, и мы на нем здесь останавливаться не будем. Мы коснемся развития теорем о больших уклонениях лишь в граничных задачах. K этому направлению Андрей Николаевич проявлял большой интерес, особенно к его качественной стороне. Первьй существенный результат в этой области также вытекает из результатов работы [1] и имеет форму неравенства для максимума $\bar{S}_{n}=\max _{k \leqslant n} S_{k}$ :

$$
\mathrm{P}\left(\bar{S}_{n}>x\right) \leqslant \frac{1}{2} \mathrm{P}\left(S_{n}>x-\sqrt{2} B_{n}\right)
$$

которое вместе с (1) и очевидным неравенством $\mathrm{P}\left(\bar{S}_{n}>x\right) \geqslant \mathrm{P}\left(S_{n}>x\right)$ немедленно дает

$$
\ln \mathrm{P}\left(\bar{S}_{n}>x\right) \sim-\frac{x^{2}}{2 B_{n}^{2}} .
$$

Эти неравенства и асимптотики (1), (2) сыграли важную роль во многих дальнейших исследованиях, например, в доказательстве компактности семейств распределений, связанных с $\left\{S_{k}\right\}$, в доказательстве принципа инвариантности и др. Сразу же отметим, что наряду с (2) интерес представляет и асимптотика "малых уклонений", т.е. вероятностей $\mathrm{P}\left(\bar{S}_{n}<x\right)$, когда $x$ и $n \rightarrow \infty$ таковы, что эта вероятность сходится к нулю.

Более обшие граничные задачи появились в работах А. Н. Колмогорова [2], [3]. Они имеют следующий вид. Будем считать для простоты, что $\xi$ и $\xi_{k}, k=1,2, \ldots$, одинаково распределены, $\mathrm{E} \xi=0, \sigma^{2}=\mathrm{E} \xi^{2}<\infty$. Тогда $B_{n}^{2}=n \sigma^{2}$. Рассмотрим две последовательности (границы) $\left\{g_{k}^{+}\right\}$и $\left\{g_{k}^{-}\right\}, g_{k}^{-}<g_{k}^{+}, k=0,1,2, \ldots$, и обозначим через $G_{x, n}$ событие

$$
G_{x, n}=\left\{x g_{k}^{-} \leqslant S_{k} \leqslant x g_{k}^{+} \text {при всех } k \leqslant n\right\} .
$$


Наиболее распространены два вида границ:

1) $g_{k}^{ \pm}$- фиксированные последовательности, не зависяшие от $n$ (как в законе повторного логарифма);

2) $g_{k}^{ \pm}=f^{ \pm}(k / n), k \leqslant n$, где $f^{ \pm}(t)$ - заданная (обычно непрерывная) функция на $[0,1]$, не зависящая от $n$.

Общая граничная задача состоит в отыскании асимптотик $\mathrm{P}\left(G_{x, n}\right)$ (или $\mathrm{P}\left(\bar{G}_{x, n}\right)$ для дополнительного к $G_{x, n}$ события $\left.\bar{G}_{x, n}\right)$ для двух названных вьше типов границ.

Задачу для второго типа границ при $x=\sqrt{n}$ (т.е. в области нормальных уклонений) назьвают часто задачей Колмогорова-Петровского. Решение этой задачи, полученное в [2], [3], состоит в следуюшем: при $x=\sqrt{n}$ и $n \rightarrow \infty$ вероятность $\mathrm{P}\left(G_{x, n}\right)$ сходится к решению уравнения теплопроводности с граничными и начальными условиями, заданными на гранище области, заключенной между кривыми $f^{ \pm}(t)$. Другими словами, $\mathrm{P}\left(G_{x, n}\right)$ сходится к вероятности $\mathrm{P}\left(f^{-}(t) \leqslant \sigma w(t) \leqslant f^{+}(t)\right.$ при всех $t \in[0,1])$, где $w(t)$ - стандартньй винеровский процесс. Этот результат подводит вплотную к принципу инвариантности Донскера-Прохорова, который был установлен лишь 20 лет спустя и который означает слабую сходимость распределений случайных ломаных $s_{n}(\cdot)$ с узлами в точках $\left(\frac{k}{n}, \frac{S_{k}}{\sigma \sqrt{n}}\right)$ к распределению винеровского процесса $w(\cdot)$. Названные фундаментальные работы А. Н. Колмогорова дают простые и ясные подходы к проблемам, которые затем обобщались во многих направлениях. Модификация этих подходов позволяет получить также и оценки скорости сходимости (см., например, [4]).

Задача о распределении $\bar{S}_{n}$ является, очевидно, частным случаем общей граничной задачи. К граничньм задачам для событий вида (3) приводят многие прикладные задачи в математической статистике (последовательньй анализ, асимптотически оптимальные процедуры в задаче разладки), в теории риска, теории массового обслуживания и др. К ним же, но в несколько измененной форме, приводят задачи, связанные с критерием Колмогорова о том, что выборка принадлежит распределению $F$. Этот критерий имеет следующий вид: названная гипотеза отвергается, если

$$
K_{x, n}=\sup _{t} \sqrt{n}\left|F_{n}^{*}(t)-F(t)\right|>x
$$

где $F_{n}^{*}$ - эмпирическая функция распределения.

Андрей Николаевич был весьма скептичен относительно практической ценности этого критерия, поскольку для многих альтернатив его мощность невелика. Он предлагал несколько иное правило

$$
K_{x, n}^{g}=\sup _{t} \frac{\sqrt{n}\left|F_{n}^{*}(t)-F(t)\right|}{g(t)}>x,
$$

где в области нормальных уклонений (вероятность ошибки 1-го рода не сходится к нулю) надо взять $g(t)=\sqrt{t(1-t)}$. Если же вероятность ошибки 1-го рода мала $(x$ велико), то вид оптимальной функции $g$ (максимизируюшей мощность) и асимптотика $\mathrm{P}\left(K_{x, n}^{g}>x\right)$ были неизвестны и представляли значительньй интерес. Эти задачи также во многом сводятся к вычислению асимптотики вероятностей событий $G_{x, n}$, 
определенных в (3). K настоящему времени осуществлено значительное продвижение в исследовании всех названных задач (см., например, обзоры в [4], [5]). Я остановлюсь кратко лишш на нескольких результатах (среди них будут и совсем недавние), которые так или иначе связаны с влиянием А.Н. Колмогорова.

Для этого я должен вновь обратиться к истории. Я начну с задачи об асимптотике $\mathrm{P}\left(\bar{S}_{n}>x\right)$, которая упоминалась первой из названных выше проблем.

Я начал заниматься этой задачей при следующих обстоятельствах. После окончания МГУ в 1954 г. я был направлен в закрытую организацию (несмотря на рекомендацию Андрея Николаевича в аспирантуру), которая занималась решением задач криптографии с помошью быстродействуюших машин. Для этих целей была создана одна из самых мошных в то время ЭВМ. В основе лежал метод перебора разных вариантов расшифрования, в результате чего на выходе появлялось большое количество $N$ вариантов расшифровки, т.е. буквенных последовательностей $a_{1}, a_{2}, \ldots, a_{n}$. Среди них надо было распознать истинньй “открытый” текст (т.е. текст английского языка, соответствующий правильному варианту расшифровки). Так как $N$ было очень велико (скажем, порядка $10^{8}-10^{10}$ ), то “вручную” это сделать было невозможно и использовался "машинный алгоритм" распознавания открытого текста. Он был основан на статистическом критерии последовательного анализа, которьй должен был различать две гипотезы: $H_{1}=\left\{\right.$ текст хаотичен, т.е. $a_{i}$ независимы, $\mathrm{P}\left(a_{i}=k\right)=$ $q(k)=1 / 26, k=1, \ldots, 26\}$ и $H_{0}=\{$ текст является открытым $\}$. В последнем случае использовались разные простейшие модели - например, $\left\{a_{i}\right\}$ считалась последовательностью независимых величин с известными вероятностями $p(k)=\mathrm{P}\left(a_{i}=k\right)$; или цепью Маркова с известными вероятностями $p_{j k}=\mathrm{P}\left(a_{i}=k / a_{i-1}=j\right)$. Близкий к оптимальному критерий в простейшем случае в силу результатов Неймана-Пирсона и Вальда выглядел следующим образом. Надо построить случайные величины $\xi_{i}=$ $\ln \frac{q\left(a_{i}\right)}{p\left(a_{i}\right)}\left(\right.$ так что $\left.\mathrm{E}\left(\xi_{i} / H_{1}\right)>0, \mathrm{E}\left(\xi_{i} / H_{0}\right)<0\right)$ и их суммы $S_{n}=\sum_{i=1}^{n} \xi_{i}$. Принимается гипотеза $H_{1}$, если $\bar{S}_{n} \geqslant x$, и $H_{0}$, если $\bar{S}_{n}<x$. Значение $x=$ const выбиралось так, чтобы вероятность ошибки 1-го рода $\mathrm{P}\left(\bar{S}_{n} \geqslant x / H_{0}\right)$ (вероятность потерять истинньй текст) имела заданное значение. Тогда $n$ должно быть настолько большим, чтобы вероятность ошибки второго рода

$$
\mathrm{P}\left(H_{0} / H_{1}\right)=\mathrm{P}\left(\bar{S}_{n}<x / H_{1}\right)
$$

имела порядок малости, скажем, $10^{-8}$. Это безусловно была задача о больших уклонениях $\bar{S}_{n}$. Я стал заниматься ею, когда обнаружил, что расчеты, по которым строился алгоритм (т.е., в частности, вычисление асимптотики $\left.\mathrm{P}\left(\bar{S}_{n}<x / H_{1}\right)\right)$, оказались ошибочными. Там были допушены даже две ошибки и они каким-то образом нивелировали друг друга, так что практический (экспериментальньй) результат не сильно отличался от теоретического. Поэтому ошибка и не была сразу замечена. В то время при поддержке Андрея Николаевича мне все же удалось поступить в заочную аспирантуру и я решил взять эту задачу в качестве диссертационной. В этом был большой риск, так как тогда по поводу этой задачи ничего не было известно, а Андрей Николаевич сразу сказал, что никаких соображений на этот счет у него нет. (Он предлагал даже взять другую задачу, но я отказался.) Риск оказался серьезным, так как почти 3 года у меня ничего не получалось. Решение для случая ограниченных решетчатых 
$\xi_{i}$ (именно такой случай и был нужен) было найдено в 1958 г. чисто аналитическим путем. Был найден явньй вид двойных преобразований над $\mathrm{P}\left(\bar{S}_{n}<x\right.$ ) (по $n$ и $x$ ) в терминах корней характеристического уравнения

$$
\mathrm{E} z^{\xi_{i}}=\mu
$$

что позволило затем с помошью теории алгебраических функций и модификаций метода перевала эти двойные преобразования обратить. При этом оказалось возможным получить полное решение задачи - найти асимптотику $\mathrm{P}\left(\bar{S}_{n}<x\right)$ и $\mathrm{P}\left(\bar{S}_{n}>x\right)$ (включая асимптотические разложения) во всем спектре уклонений [6].

Позже эти результаты были обобшены и на случай произвольно распределенных $\xi_{i}$ с помошью редукции проблемы к граничным задачам теории функций комплексного переменного (к задаче Римана-Гильберта о факторизации функций) [7]. Эти задачи возникают при решении уравнений типа Винера-Хопфа, которые описьвают производящую функщию распределения $\bar{S}_{n}$; к тому времени эти уравнения были достаточно хорошо изучены.

Здесь следует отметить следуюшее. Андрей Николаевич проявил большой интерес к тому, как здесь развивались события (особьй интерес он проявлял почему-то к форме и смыслу коэффициентов асимптотических разложений). Однако он постоянно выражал некоторое "неудовольствие" тем, что решение задачи было чисто аналитическим и что не было найдено достаточно прозрачных, прямых, вероятностных подходов к этому решению. Об этом его "неудовольствии" я помнил все время, и лишь сравнительно недавно (спустя почти 40 лет) такой более простой вероятностный подход найти удалось. Он тоже во многом является аналитическим, но все же намного проще и прозрачнее. Я на нем кратко остановлюсь. Мы будем предполагать, что преобразование Лапласа

$$
\varphi(\lambda)=\mathrm{E} e^{\lambda \xi}
$$

аналитично в окрестности точки $\lambda(0)$, в которой достигается $\min _{\lambda} \varphi(\lambda) \leqslant 1$. Через $\Lambda(\alpha)$ обозначим функцию уклонений (преобразование Лежандра)

$$
\Lambda(\alpha)=\sup _{\lambda}(\lambda \alpha-\ln \varphi(\lambda))
$$

и через $\lambda(\alpha)=\Lambda^{\prime}(\alpha)$ - точку, где этот sup достигается.

Преобразованием Крамера с параметром $\alpha$ над распределением $\xi$ мы будем называть распределение $P^{(\alpha)}$,

$$
P^{(\alpha)}(d x)=\mathrm{P}\left(\xi^{(\alpha)} \in d x\right)=\frac{\mathrm{P}(\xi \in d x) e^{\lambda x}}{\varphi(\lambda)} \text { при } \lambda=\lambda(\alpha),
$$

и обозначать через $\xi^{(\alpha)}$ соответствующую случайную величину. Нетрудно проверить, что $\mathrm{E} \xi^{(\alpha)}=\alpha$. Для простоты ограничимся в дальнейшем решетчатым случаем, считая, что $\xi$ целочисленна с н.о.д. возможных значений равньм 1.

Новый подход к граничньм задачам был связан со следуюшими фактами. 
1) Распределение $P^{(\alpha)}$ есть предельное условное распределение скачков $\xi_{k}$ при условии $S_{n}=x, \frac{x}{n} \rightarrow \alpha$ : для любых $k \leqslant n$ и борелевского множества $B$

$$
\lim _{n \rightarrow \infty} \mathrm{P}\left(\xi_{k} \in B / S_{n}=x\right)=P^{(\alpha)}(B)
$$

2) Для любого набора $k_{1}, \ldots, k_{l}, 1 \leqslant k_{1}<\cdots<k_{l} \leqslant n$, скачки $\xi_{k_{1}}, \ldots, \xi_{k_{l}}$ при условии $S_{n}=x, x / n \rightarrow \alpha$, асимптотически независимы:

$$
\lim _{n \rightarrow \infty} \mathrm{P}\left(\bigcap_{i=1}^{l}\left\{\xi_{k_{i}} \in B_{i}\right\} / S_{n}=x\right)=\prod_{i=i}^{l} P^{(\alpha)}\left(B_{i}\right) .
$$

Для условной траектории $\left\{S_{k}\right\}$ сохраняются также многие основные предельные теоремы (закон повторного логарифма, сходимость к броуновскому мосту и др.) [8].

Отсюда нетрудно извлечь следуюшее. Пусть в нашей задаче $\mathrm{E} \xi>0$ (как в приведенном выше примере с вычислением $\left.\mathrm{P}\left(\bar{S}_{n}<x / H_{1}\right)\right), \frac{x}{n} \rightarrow \alpha<\mathrm{E} \xi$. Тогда

$$
\mathrm{P}\left(\bar{S}_{n}<x\right)=\sum_{j=-\infty}^{x-1} \mathrm{P}\left(S_{n}=j\right) \mathrm{P}\left(\bar{S}_{n}<x / S_{n}=j\right)
$$

Здесь асимптотика $\mathrm{P}\left(S_{n}=j\right)$ хорошо изучена (см. ниже), а вероятность

$$
\mathrm{P}\left(\bar{S}_{n}<x / S_{n}=j\right)
$$

в силу сказанного выше при больших $n$ близка к вероятности

$$
\mathrm{P}\left(\max _{k>0}\left(-S_{k}^{(\alpha)}\right)<x-j\right)=p(x-j)
$$

(траектория, стартующая в обратном во времени направлении из точки $(n, j)$, при каждом фиксированном $x-j$, имеет скачки с распределением, близким к распределению $-\xi^{(\alpha)}$, и состоит из “почти” независимых скачков). Вероятности $p(y)$ могут быть найдены в терминах компонент факторизации (или в терминах нулей характеристического уравнения (4) в нашем примере). Поскольку при $x-j=o(n)$

$$
\begin{gathered}
\mathrm{P}\left(S_{n}=j\right) \sim \frac{e^{-n \Lambda(j / n)}}{\sqrt{2 \pi n} \sigma^{(\alpha)}}, \\
\text { где }\left(\sigma^{(\alpha)}\right)^{2}=\mathrm{D}\left(\xi^{(\alpha)}\right)^{2}, n \Lambda(j / n)=n \Lambda\left(\frac{x}{n}\right)+(x-j) \lambda\left(\frac{x}{n}\right)+o(x-j), \text { то } \\
\mathrm{P}\left(\bar{S}_{n}<x\right) \sim \frac{e^{-n \Lambda(x / n)}}{\sigma^{(\alpha)} \sqrt{2 \pi n}} \sum_{j=-\infty}^{x-1} p(x-j) e^{\lambda(\alpha)(x-j)},
\end{gathered}
$$


где $\lambda(\alpha)<0$ при $\alpha<\mathrm{E} \xi>0, p(k) \rightarrow 1$ при $k \rightarrow \infty$, так что $\sum_{k=1}^{\infty} p(k) e^{k \lambda(\alpha)} \equiv$ $c(\alpha)<\infty$,

$$
\mathrm{P}\left(\bar{S}_{n}<x\right) \sim \frac{c(\alpha) e^{-n \Lambda(x / n)}}{\sigma^{(\alpha)} \sqrt{2 \pi n}} \sim c(\alpha) \mathrm{P}\left(S_{n}=x\right) \sim \frac{c(\alpha) \mathrm{P}\left(S_{n}<x\right)}{1-e^{\lambda(\alpha)}}
$$

В нашем примере с $\mathrm{P}\left(\bar{S}_{n}<x / H_{1}\right)$, где $x$ фиксировано, надо положить $\alpha=0$.

Аналогичным образом находится асимптотика $\mathrm{P}\left(\bar{S}_{n}>x\right)$, если $x / n \rightarrow \alpha>\mathrm{E} \xi$.

Если $\mathrm{E} \xi<0, x \rightarrow \infty$, то для вычисления $\mathrm{P}\left(\bar{S}_{n}>x\right)$ можно использовать тот же способ подсчета. При $n>(c+\varepsilon) x$ будет выполняться

$$
\mathrm{P}\left(\bar{S}_{n}>x\right) \sim q e^{-\beta x}
$$

где $q=$ const $\in(0,1), \beta>0$ - решение уравнения $\varphi(\lambda)=1$.

Таким образом, во всех названных задачах роль факторизации сводится к влиянию лишь на постоянньй множитель. Сама асимптотика (с точностью до этого множителя) от компонент факторизации не зависит.

Важнейшее отличие описанного подхода от упомянутого вьше аналитического состоит в том, что

1) он легко переносится на криволинейные границы, когда изучается, скажем (ср. c (3)),

$$
\mathrm{P}\left(\max _{k \leqslant n}\left(S_{k}-x g_{k}\right)>0\right)
$$

где $x g_{n}>n \mathrm{E} \xi$, граница $\left\{g_{k} \neq\right.$ const $\}$ “дифференцируема" в точке $n$, т.е. при каждом $j$ и $n \rightarrow \infty$

$$
g_{n}-g_{n-j} \sim g j, \quad g<\frac{x g_{n}}{n}(1-\varepsilon), \quad \varepsilon>0
$$

2) он легко переносится на многомерный случай [9].

Таким образом, общая граничная задача, скажем, об асимптотике вероятности события $\bar{G}_{n, x}$, дополнительного к $(3)$, в случае, когда полоса между $g_{k}^{ \pm}$является “широкой":

$$
\mathrm{P}\left(\bar{G}_{n, x}\right) \sim \mathrm{P}\left(\max _{k \leqslant n}\left(S_{k}-x g_{k}^{+}\right)>0\right)+\mathrm{P}\left(\min _{k \leqslant n}\left(S_{k}-x g_{k}^{-}\right)<0\right)
$$

может быть решена теперь в области больших уклонений (когда оба слагаемых в правой части (5) сходятся к нулю) в явном виде при весьма широких предположениях. При этом новьй подход во многом является прямым вероятностным, допускаюшим естественное распространение на многомерный случай.

В случае нерешетчатых $\xi$ все вьглядит аналогичным образом. Надо лишь событие $\left\{S_{n}=x\right\}$ в условных вероятностях заменить на $\left\{S_{n} \in \Delta(x)\right\}$, где $\Delta(x)$ - интервал $\Delta(x)=(x, x+\Delta]$ при фиксированном $\Delta>0$.

Случай, когда полосав событии $(3)$ является “узкой”, т.е. $\mathrm{P}\left(G_{n, x}\right) \rightarrow 0$, был изучен в [10] (см. также обзор в [5]). 


\section{2. О распределении максимума сумм при невыполнении условия Крамера}

Я остановлюсь теперь на задаче о распределении $\bar{S}_{n}$ в случае, когда условие Крамеpa (о конечности $\varphi(\lambda)$ при некотором $\lambda>0)$ не вьполнено и мы имеем дело с так называемыми "толстыми хвостами" $\mathrm{P}(\xi>x)$, убывающими медленнее, чем любая экспонента. В 60-е годы граничные задачи с такими хвостами скачков не привлекали большого внимания. Однако в течение последних 10-20 лет они стали очень популярными. Причина состоит в том, что в ряде приложений, например, в теории страхования, такие хвосты представляют наибольший интерес. Рассмотрим простейшую модель с дискретным временем поведения капитала страховой компании. Пусть $x$ - начальный капитал и $\xi_{k}$ - убыль капитала за $k$-й день работы компании. Тогда если допускать и отрицательное значение капитала, то $x+S_{n}$ будет означать капитал компании после $n$ дней работы. Убыль капитала $\xi_{k}$ имеет вид $\xi_{k}=\zeta_{k}-v_{k}$, где $\zeta_{k} \geqslant 0$ - выплаты пострадавшим клиентам, $v_{k} \geqslant 0$ - страховые взносы. Вьплаты и взносы подбирают таким образом, чтобы в соответствии с существующей статистикой выполнялось $\mathrm{E} \xi<0$ (если $\left.\mathrm{E} \zeta_{k}<\infty\right)$. Очевидно, что вероятность того, что компания разорится в течение времени $n$, равна $\mathrm{P}\left(\bar{S}_{n}>x\right)$.

Давно было замечено, что большие вьплаты больших страховых компаний, скажем, в размере 200 млн. долларов, случаются примерно лишь вдвое реже, чем выплаты в 100 млн. долларов. Это означает, грубо говоря, что хвост $\mathrm{P}\left(\zeta_{k}>x\right)$ (а стало быть, и $\left.\mathrm{P}\left(\xi_{k}>x\right)\right)$ ведет себя при $x \rightarrow \infty$ как $\frac{L(x)}{x}$, где $L$ - медленно меняюшаяся функция (м.м.ф.).

Рассмотрим сначала класс $\mathscr{R}$ хвостов вида

$$
\mathrm{P}(\xi>x)=V(x)=x^{-\beta} L(x), \quad \beta>0,
$$

где выделим две возможности:

1) $a=\mathrm{E} \xi$ сушествует,

2) $\mathrm{E}|\xi|=\infty$.

В первом случае $\bar{S}=\bar{S}_{\infty}<\infty$ п.н. тогда и только тогда, когда $a<0$. Во втором случае условие конечности $\bar{S}$ имеют более сложньй вид (см. ниже). В обоих случаях основной интерес представляет асимптотика $\mathrm{P}(\bar{S}>x)$. В [11] для класса $\mathscr{R}$ и некоторых его расширений было установлено, что при $a<0$

$$
\mathrm{P}(\bar{S}>x) \sim \widetilde{V}(x) \equiv \frac{1}{|a|} \int_{x}^{\infty} V(u) d u
$$

В [12] этот результат был распространен на класс $\mathscr{S}$ субэкспоненциальных распределений.

Распределение $G$ с хвостом $G(x)=G((\infty, \infty))$ называется субәкспоненциальным, если оно локально постоянно:

$$
\frac{G(x+v)}{G(x)} \rightarrow 1 \text { при любом фиксированном } v \text { и } x \rightarrow \infty,
$$


и его свертка $G^{* 2}(x)=\int G(d t) G(x-t)$ обладает свойством

$$
\frac{G^{* 2}(x)}{G(x)} \rightarrow 2 \text { при } x \rightarrow \infty .
$$

Оказьвается, что $\mathscr{S}$ есть максимальный класс функций, для которого вьполнено (7) (т.е. (7) влечет за собой $V \in \mathscr{S}[13])$.

Недавно в [14], [15] было установлено, что для некоторых классов хвостов, близких к $\mathscr{S}$, выполнено также

$$
\mathrm{P}\left(\bar{S}_{n}>x\right) \sim \frac{1}{|a|} \int_{x}^{x-a n} V(u) d u .
$$

В связи со значительной ролью, которую играет класс субэкспоненциальных распределений $\mathscr{S}$ во многих исследованиях, возникает естественный вопрос о характеризации этого класса. Для сравнений нам понадобится класс $\mathscr{S} e$ так называемых семиәкспоненииальных распределений, которьй описьвается "явньм образом": $V \in \mathscr{S} e$, если

$$
V(x)=e^{-l(x)}, \quad l(x)=x^{\alpha} L(x), \quad \alpha \in[0,1], \quad L-\text { м.м.ф. },
$$

где $l$ обладает свойствами: $l(x)=o(x)$,

$$
l(x+\Delta)-l(x) \sim \frac{\alpha \Delta l(x)}{x},
$$

если $\frac{\Delta l(x)}{x}>\varepsilon$ при каком-нибудь $\varepsilon>0, x \rightarrow \infty, \Delta=o(x)$, и

$$
l(x+\Delta)-l(x)=o(1)
$$

если $\frac{\Delta l(x)}{x} \rightarrow 0$ при $x \rightarrow \infty$.

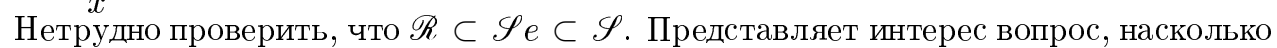
класс $\mathscr{S}$ шире $\mathscr{S}$ e. В известной мере, ответ на него, а также характеризация класса $\mathscr{S}$, содержатся в следующих утверждениях.

Обозначим

$$
r(k)=\frac{l(k+1)-l(k)}{l(k)} .
$$

Нетрудно видеть, что для $G \in \mathscr{S}$ существует $\lim _{k \rightarrow \infty} k r(k)=\beta \leqslant 1$.

Tеорема 1. 1) Eсли $G(x)=e^{-l(x)}$ локально постоянно и

$$
\alpha^{+}=\varlimsup_{k \rightarrow \infty} k r(k)<1
$$

mo $G \in \mathscr{S}$.

2) $\operatorname{Ecлu~} G \in \mathscr{S}$, mo

$$
\alpha^{-}=\underline{\lim } k r(k) \leqslant 1 \text {. }
$$


3) $\operatorname{Ecлu~} G \in \mathscr{S}$ u $\alpha^{+}=\alpha^{-}=\alpha$, mo $G \in \mathscr{S} e$.

Если $G(t)=e^{-l(t)} \in \mathscr{S}(G \in \mathscr{S} e)$, то всегда сушествует функция $G_{1}(t)=e^{-l_{1}(t)}$ такая, что $l_{1}$ дифференцируема, $l_{1}(t)=l(t)+o(1)\left(G_{1}(t) \sim G(t)\right.$ при $\left.t \rightarrow \infty\right), G_{1} \in \mathscr{S}$ $\left(G_{1} \in \mathscr{S} e\right)$. Можно взять, например, $l_{1}(t)=\int_{t}^{t+1} l(u) d u$. Таким образом, не ограничивая общности, можно считать, что $l$ дифференщируема. Тогда вместо асимптотической характеристики $r(k)$ в теореме 1 можно взять асимптотическую характеристику $(\ln l(t))^{\prime}$ и положить

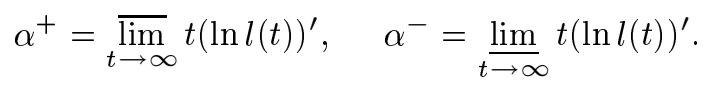

Итак, теорема 1 показьвает, что класс $\mathscr{S}$ сушественно шире, чем $\mathscr{S} e$, а $\mathscr{S} e$ можно представить себе как "регулярную" $\left(\alpha^{+}=\alpha^{-}\right)$часть $\mathscr{S}$. С помощью теоремы нетрудно проверить, например, что следующие довольно сильные отклонения от функций $G_{0}(x)=e^{-t^{\alpha}} \in \mathscr{S} e$ вновь вместе с $G_{0}$ принадлежат $\mathscr{S}$. Пусть $l_{0}(t)=t^{\alpha}, \alpha \in(0,1)$, $t_{k}=2^{k}, \delta<1$. Тогда функция $l(t)$, построенная по формуле

$$
l(t)= \begin{cases}l_{0}\left(t_{k}\right)=\mathrm{const} & \text { при } t_{k}<t \leqslant(1+\delta) t_{k}, \\ l_{0}\left(t_{k}\right)+t^{\alpha}-\left((1+\delta) t_{k}\right)^{\alpha} & \text { при }(1+\delta) t_{k} \leqslant t \leqslant t_{k+1},\end{cases}
$$

будет соответствовать распределению $G=e^{-l} \in \mathscr{S}$. Яно, что здесь $G \notin \mathscr{S} e, \alpha^{+}=$ $\alpha \neq \alpha^{-}=0$.

При более детальном изучении вероятностей больших уклонений $S_{n}$ и $\bar{S}_{n}$ классы $\mathscr{R}$ и $\mathscr{S} e$ оказьваются все же более удобными, чем класс $\mathscr{S}$, и позволяют получать более “продвинутые” результаты (см., например, [15], [16] и библиографию там). Это относится и к распределению $\bar{S}$, для которого в классах $\mathscr{R}$ и $\mathscr{S} e$ можно получать асимптотические разложения.

Теорема 2. Пусть $\mathrm{E} \xi<0, \mathrm{E} \xi^{2}<\infty, V \in \mathscr{R}$ или $V \in \mathscr{S e}$, распределение $\xi$ нерешетчато. Тогда

$$
\mathrm{P}(\bar{S}>x)=\tilde{V}(x)+c V(x)+o(V(x))
$$

əде $c=\frac{\mathrm{E} \xi^{2}}{2(\mathrm{E} \xi)^{2}}-\frac{\mathrm{E} \bar{S}}{\mathrm{E} \xi}$.

Такое же утверждение справедливо для решетчатых $\xi$ (при х, кратных шагу решетки), но постоянная с будет несколько иной.

Отсюда, в частности, следует, что для интервалов $\Delta(x)=(x, x+\Delta)$ вьполняется

$$
\mathrm{P}(\bar{S} \in \Delta(x)) \sim \frac{\Delta}{|a|} V(x) .
$$

Это последнее утверждение было получено недавно [17], [18] для более широких классов распределений, близких к $\mathscr{S}$.

Ситуация сушественно изменится, если $\mathrm{E}|\xi|=\infty$. Тогда сушественную роль в описании асимптотики $\mathrm{P}(\bar{S}>x)$ начинает играть левьй хвост $W(t)=\mathrm{P}(\xi<-t), t>0$. Пусть оба хвоста принадлежат $\mathscr{R}$ (т.е. вьполнено (6) и $W(t)=t^{-\beta_{W}} L_{W}(t), \beta_{W}>0$ ). 
Tеорема $3[19] . \bar{S}<\infty$ тогда и только тогда, когда

$$
\int_{1}^{\infty} V\left(W^{(-1)}\left(\frac{1}{t}\right)\right) d t<\infty .
$$

Грубо говоря, этот интеграл сходится, если $V(t)<W(t)(\ln t)^{-1-\varepsilon}, \varepsilon>0$, и расходится, если $V(t)>W(t)(\ln t)^{-1}$. В частности, интеграл (8) всегда сходится, если $\beta_{W}<\beta$. Критерий конечности $\bar{S}$ в общем случае (без наличия регулярных мажорант или минорант для хвостов $\xi$ ) получен в [20].

В случае $\beta_{W}<\beta$ удается также найти асимптотику $\mathrm{P}(\bar{S}>x)$.

Tеорема 4 [19]. Eсли $\beta_{W}<\beta$, то при $x \rightarrow \infty$

$$
\mathrm{P}(\bar{S}>x) \sim \frac{V(x)}{W(x)} .
$$

Это соотношение дает, вообще говоря, иную асимптотику, чем $c x V(x)$, вытекающую из (7), но "непрерьвно" переходящую в нее при "граничном" значении $\beta_{W}=1$.

Изучение распределения $S_{n}$ и асимптотики событий $\bar{G}_{x, n}$ (см. (3) и общую граничную задачу) для классов распределений $\mathscr{R}$ и $\mathscr{S} e$ происходит значительно проще, чем в крамеровском случае (см., например, [15], [16] и библиографию там).

\section{СПИСОК ЛИТЕРАТУРЫ}

[1] A. N. Kolmogoroff. Über das Gesetz des iterierten Logarithmus // Math. Ann. 1929. V. 101. №1. Р. 126-135. Рус. пер.: О законе повторного логарифма // А.Н. Колмогоров. Теория вероятностей и математическая статистика. М.: Наука, 1986. С. 34-44.

[2] A. N. Kolmogoroff. Über die analytischen Methoden in der Wahrschenlichkeitsrechnung // Math. Ann. 1931. V. 104. Р. 415-458. Рус. пер.: Об аналитических методах в теории вероятностей // УМН. 1938. № 5. С. 5-41; А.Н. Колмогоров. Теория вероятностей и математическая статистика. М.: Наука, 1986. С. 60-105.

[3] A. N. Kolmogoroff. Über die Grenzwertsätze der Wahrschenlichkeitsrechnung // Изв. АН СССР. ОМЕН. 1933. № 3. С. 363-372. Рус. пер.: О предельных теоремах теории вероятностей // А. Н. Колмогоров. Теория вероятностей и математическая статистика. М.: Наука, 1986. C. $141-148$.

[4] А.А. Боровков. Граничные задачи, принцип инвариантности, большие уклонения // УМH. 1983. Т. 38. № 4. C. 227-254.

[5] А. А. Боровков, А. А. Могульский, А. И. Саханенко. Предельные теоремы для случайных процессов // Итоги науки и техники. Совр. проблемы матем. Фунд. напр. Т. 82. М.: ВИНИТИ, 1995.

[6] А. А. Боровков. Предельные теоремы о распределении максимума сумм ограниченных решетчатых случайных величин. I, II // Теория вероятн. и ее примен. 1960. Т. 5. № 2. C. $137-171 ;$ 1960. T. 5. № 4. C. 377-392.

[7] А. А. Боровков. Новые предельные теоремы в граничных задачах для сумм независимых слагаемых // Сиб. матем. журн. 1962. Т. 3. № 5. С. 645-694.

[8] А.А. Боровков. Об условных распределениях, связанных с болшшими уклонениями // Сиб. матем. журн. 1996. Т. 37. № 4. С. 732-744.

[9] А. А. Боровков, А. А. Могульский. Предельные теоремы в задаче достижения границы многомерным блужданием // Сиб. матем. журн. 2001. Т. 42. № 2. С. 289-317.

[10] А. А. Могульский. Вероятности больших уклонений для случайных блужданий // Труды Ин-та математики СО АН СССР. 1983. Т. 3. С. 93-124. 
[11] А. А. Боровков. Вероятностные процессы в теории массового обслуживания. М.: Физматгиз, 1972.

[12] N. Veraverbeke. Asymptotic behaviour of Wiener-Hopf factors of a random walks // Stochastic Process. Appl. 1977. V. 5. № 1. P. 27-37.

[13] D. A. Korshunov. On distribution tail of the maximum of a random walk // Stochastic Process. Appl. 1997. V. 72. № 1. P. 97-103.

[14] Д. А. Коршунов. Вероятности больших уклонений максимумов сумм независимых слагаемых с отрицательньм средним и субэкспоненциальным распределением // Теория вероятн. и ее примен. 2001. Т. 46. №2. С. 387-397.

[15] А. А. Боровков. Вероятности больших уклонений для случайных блужданий с семиэкспоненциальными распределениями // Сиб. матем. журн. 2000. Т. 41. № 6. С. 1290-1324.

[16] А. А. Боровков, К.А. Боровков. О вероятностях больших уклонений случайных блужданий. I: Распределения с правильно меняющимися хвостами // Теория вероятн. и ее примен. 2001. Т. 46. № 2. С. 209-232.

[17] S. Asmussen, V. Kalashnikov, D. Konstantinides, C. Klüppelberg, G. Tsitsiashvili. A local limit theorem for random walk maxima with heavy tails // Statist. Probab. Lett. 2002. V. 56. № 4. P. 399-404.

[18] S. Asmussen, S. G. Foss, D. A. Korshunov. Asymptotics for sums of random variables with local subexponential behaviour // J. Theoret. Probab. 2003. V. 16. № 2. P. 489-518.

[19] A. A. Borovkov. Large deviations probabilities for random walks in the absence of finite expectations of jumps // Probab. Theory Related Fields. 2003. V. 125. № 3. P. 421-446.

[20] K. B. Erickson. The strong law of large numbers when the mean is undefined // Trans. Amer. Math. Soc. 1973. V. 185. P. 371-381.

Институт математики им. С. Л. Соболева СО РАН

Поступила в редакцию

E-mail: borovkov@math.nsc.ru

18.06 .2003 